PROCEEDINGS OF THE AMERICAN MATHEMATICAL SOCIETY

Volume 124, Number 8, August 1996

\title{
SOME CONVERSES OF THE STRONG SEPARATION THEOREM
}

\author{
HWA-LONG GAU AND NGAI-CHING WONG
}

(Communicated by Dale Alspach)

To the memory of Yau-Chuen Wong (1935.10.2 - 1994.11.7)

\begin{abstract}
A convex subset $B$ of a real locally convex space $X$ is said to have the separation property if it can be separated from every closed convex subset $A$ of $X$, which is disjoint from $B$, by a closed hyperplane. The strong separation theorem says that if $B$ is weakly compact, then it has the separation property. In this paper, we present two versions of the converse and discuss an application of them. For example, we prove that a normed space is reflexive if and only if its closed unit ball has the separation property. Results in this paper can be considered as supplements of the famous theorem of James.
\end{abstract}

\section{INTRODUCTION}

Let $B$ be a bounded convex subset of a real locally convex (Hausdorff) space $X$. $B$ is said to have the separation property if it can be strictly separated from every closed convex subset $A$ of $X$, which is disjoint from $B$, by a closed hyperplane, i.e. there is a continuous linear functional $f$ of $X$ such that

$$
\inf \{f(x): x \in A\}>\sup \{f(x): x \in B\} .
$$

$B$ is said to have James' property if every continuous linear functional $f$ of $X$ attains its supremum on $B$, i.e. there is a $b$ in $B$ such that

$$
f(b)=\sup \{f(x): x \in B\} .
$$

The classical strong separation theorem of Klee [7] states that if $B$ is weakly compact, then $B$ has the separation property. It is also plain that if $B$ has the separation property, then $B$ has James' property. In this paper, we shall investigate possible converses of the above two implications.

In a series of papers $[2,3,4,5,6]$, R.C. James established the famous James' Theorem which was extended by J. D. Pryce [8] as

James' Theorem. For a complete bounded convex subset $B$ of a locally convex space $X, B$ is weakly compact if and only if $B$ has James' property.

A major application of James' Theorem is a characterization of the reflexivity of Banach spaces: A Banach space $E$ is reflexive if and only if the closed unit ball of $E$ has James' property.

Received by the editors October 18, 1994 and, in revised form, February 22, 1995.

1991 Mathematics Subject Classification. Primary 46A03, 46A25, 46B10.

This research is partially supported by National Science Council of Taiwan, R.O.C.

(C)1996 American Mathematical Society 
James' Theorem cannot be extended further to incomplete bounded convex sets. In [5], R.C. James presented a counterexample to show that a bounded convex set with James' property is not necessarily weakly compact even if it is the closed unit ball of a normed space. We shall use the same example to show that a bounded convex set with James' property does not necessarily have the separation property, either (see Example 6). In other words, the separation property is closer to weak compactness than James' property in general. As evidence, we obtain

Theorem 1. A bounded convex body (i.e. convex set with nonempty interior) $B$ in a real normed space $X$ is weakly compact if and only if $B$ has the separation property. In particular, a real normed space is reflexive if and only if its closed unit ball has the separation property.

Conjecture. A bounded convex subset $B$ of a real locally convex space $X$ is weakly compact if and only if $B$ has the separation property.

We shall present in Theorem 3 a sufficient condition under which our conjecture holds. Theorem 7 demonstrates an application of our results. It shows clearly that even a partial answer of our conjecture can improve many classical results, in particular, for those involving completeness conditions. Although we discuss only real locally convex spaces in this paper, our results should be easily extended to complex cases.

We would like to take this opportunity to express our great gratitude to Louis de Branges and Yau-chuen Wong for their kind motivation and encouragement.

\section{Main Results}

In the following, $B$ always denotes a bounded convex subset of a real locally convex space $X$. We note that if $B$ has the separation property, then $B$ is weakly closed. It is clear that Theorem 1 is a corollary of James' Theorem and the following lemma.

Lemma 2. Let $B$ be a bounded convex body in a real normed space $X$. If $B$ has the separation property, then $B$ is complete.

Proof. Without loss of generality, we can assume $0 \in B$. Let $\tilde{B}$ be the closure of $B$ in the completion $\tilde{X}$ of $X$. Note that $\tilde{B}$ is a bounded convex body in $\tilde{X}$. For any nonzero $b$ in $\tilde{B}, \lambda b$ belongs to the boundary of $\tilde{B}$, where $\lambda=\sup \{k: k b \in \tilde{B}\} \geq 1$. We want to show that $\tilde{B}=B$. It suffices to verify that $B$ contains the boundary of $\tilde{B}$.

Suppose there were an element $b$ in the boundary of $\tilde{B}$ such that $b \notin B$. Let $b$ be contained in a supporting hyperplane $H$ of $\tilde{B}$ such that $H=\{x \in \tilde{X}: f(x)=1\}$ and $\tilde{B} \subset\{x \in \tilde{X}: f(x) \leq 1\}$ for some continuous linear functional $f$ of $\tilde{X}$. In particular, $f(b)=1$. Let $b_{n}=\left(1+\frac{1}{n}\right) b$ for $n=1,2,3, \cdots$. Let $B_{\tilde{X}}(a ; \delta)$ denote the open ball $\{x \in \tilde{X}:\|x-a\|<\delta\}$. Since $B_{\tilde{X}}\left(b_{n} ; \frac{1}{n}\right) \cap\left\{x \in \tilde{X}: f(x)>1+\frac{1}{n}\right\}$ is non-empty and open in $\tilde{X}$ for each $n=1,2, \cdots$ and $X$ is dense in $\tilde{X}$, we can choose $a_{n}$ 's from $X$ so that $a_{n} \in B_{\tilde{X}}\left(b_{n} ; \frac{1}{n}\right) \cap\left\{x \in \tilde{X}: f(x)>1+\frac{1}{n}\right\}$. Then $f\left(a_{n}\right)>1+\frac{1}{n}$ for $n=1,2,3, \cdots$, and the sequence $\left(a_{n}\right)$ converges to $b$ in norm.

Let $A$ be the closed convex hull of the $a_{n}$ 's in $X$. We want to show that $A \cap B=\emptyset$. Suppose an element $y$ in $X$ exists such that $y \in A \cap B$. Note that $y \neq b$ since $b \notin B$. Let $N$ be a positive integer such that $B_{\tilde{X}}\left(b ; \frac{2}{N}\right) \cap B_{\tilde{X}}\left(y ; \frac{2}{N}\right)=\emptyset$. Since $y \in A$, there exists a sequence $\left\{y_{n}\right\}$ of convex combinations of $a_{n}$ 's converging to $y$ in norm. For 
each $n$, write $y_{n}=\sum_{i=1}^{k_{n}} \alpha_{i}^{n} a_{i}$, where $\alpha_{i}^{n} \geq 0$ for $i=1,2, \cdots, k_{n}, \sum_{i=1}^{k_{n}} \alpha_{i}^{n}=1$, and $k_{n}$ is a positive integer depending on $n$. Since $y_{n} \rightarrow y$ in norm and $f(y)=1$, there exists a positive integer $M_{1}$ such that $f\left(y_{n}\right)<1+\frac{1}{N}$ for all $n \geq M_{1}$. For each $n \geq M_{1}, 1+\frac{1}{N}>f\left(\sum_{i=1}^{k_{n}} \alpha_{i}^{n} a_{i}\right)=\sum_{i=1}^{k_{n}} \alpha_{i}^{n} f\left(a_{i}\right)>\sum_{i=1}^{k_{n}} \alpha_{i}^{n}\left(1+\frac{1}{i}\right)=1+\sum_{i=1}^{k_{n}} \frac{\alpha_{i}^{n}}{i}$. This implies $\sum_{i=1}^{k_{n}} \frac{\alpha_{i}^{n}}{i}<\frac{1}{N}$. On the other hand, there exists a positive integer $M_{2}$ such that $y_{n} \in B_{\tilde{X}}\left(y ; \frac{2}{N}\right), \forall n \geq M_{2}$. For $n \geq M=\max \left\{M_{1}, M_{2}\right\},\left\|y_{n}-b\right\|=$ $\left\|\sum_{i=1}^{k_{n}} \alpha_{i}^{n} a_{i}-b\right\|=\left\|\sum_{i=1}^{k_{n}} \alpha_{i}^{n}\left(a_{i}-b\right)\right\| \leq \sum_{i=1}^{k_{n}} \alpha_{i}^{n}\left\|a_{i}-b\right\|<2 \sum_{i=1}^{k_{n}} \frac{\alpha_{i}^{n}}{i}<\frac{2}{N}$. This implies $y_{n} \in B_{\tilde{X}}\left(b ; \frac{2}{N}\right), \forall n \geq M$. This contradicts the fact that $B_{\tilde{X}}\left(b ; \frac{2}{N}\right) \cap$ $B_{\tilde{X}}\left(y ; \frac{2}{N}\right)=\emptyset$. Hence $A \cap B=\emptyset$.

By the separation property of $B$, there is a continuous linear functional $g$ of $X$ such that

$$
\sup \{g(u): u \in B\}<\inf \{g(a): a \in A\}
$$

Let $g^{\prime}$ be a continuous extension of $g$ to $\tilde{X}$. Since $a_{n} \rightarrow b$ as $n \rightarrow \infty$ in $\tilde{X}$,

$$
g^{\prime}(b)=\lim _{n \rightarrow \infty} g\left(a_{n}\right) \geq \inf \{g(a): a \in A\}>\sup \{g(u): u \in B\} \geq g^{\prime}(b) .
$$

This is a contradiction! Therefore $B=\tilde{B}$, and thus $B$ is complete.

Let $(X, \Im)$ be a locally convex space. A subset $B$ of $X$ is said to be absolutely convex if $\lambda a+\beta b \in B$ whenever $a, b \in B$ and $|\lambda|+|\beta| \leq 1$. For any absolutely convex $\Im$-bounded subset $B$ of $X$, let $X(B)$ be the linear span of $B$. Then $X(B)=$ $\bigcup_{n} n B$, and $B$ is absorbing in $X(B)$. Hence the gauge $\gamma_{B}$ of $B$, defined by

$$
\gamma_{B}(x)=\inf \{\lambda>0: x \in \lambda B\}
$$

is a seminorm on $X(B)$ and

$$
\left\{x \in X(B): \gamma_{B}(x)<1\right\} \subset B \subset\left\{x \in X(B): \gamma_{B}(x) \leq 1\right\} .
$$

Moreover, the boundedness of $B$ ensures that $\left.\Im\right|_{X(B)}$ (the relative topology induced by $\Im)$ is coarser than the $\gamma_{B}(\cdot)$-topology. Thus, $\gamma_{B}$ is actually a norm on $X(B)$.

Theorem 3. Let $B$ be a bounded absolutely convex subset of a real locally convex space $(X, \Im)$ such that $\left(X(B), \gamma_{B}\right)^{*}=\left(X(B),\left.\Im\right|_{X(B)}\right)^{*}$. Then $B$ is weakly compact if and only if $B$ has the separation property.

Proof. The necessity is clear. For the sufficiency, we shall show that every closed and bounded convex subset $A$ of $\left(X(B), \gamma_{B}\right)$, which is disjoint from $B$, can be strictly separated from the closed unit ball $B$ of $\left(X(B), \gamma_{B}\right)$. Since the $\gamma_{B}(\cdot)$-topology is consistent with the duality $\left\langle\left(X(B),\left.\Im\right|_{X(B)}\right),\left(X(B),\left.\Im\right|_{X(B)}\right)^{*}\right\rangle, A$ is also a closed convex subset of $\left(X(B),\left.\Im\right|_{X(B)}\right)$. By the boundedness of $A$ in $\left(X(B), \gamma_{B}\right), A$ is closed in $(X, \Im)$. The separation property of $B$ provides an $f$ in $(X, \Im) *$ such that

$$
\sup \{f(b): b \in B\}<\inf \{f(a): a \in A\} .
$$

Let $g=\left.f\right|_{X(B)}$; then $g \in\left(X(B),\left.\Im\right|_{X(B)}\right)^{*}=\left(X(B), \gamma_{B}\right)^{*}$ and

$$
\sup \{g(b): b \in B\}<\inf \{g(a): a \in A\} .
$$

Therefore, the closed unit ball $B$ of $\left(X(B), \gamma_{B}\right)$ has the separation property, too. By Theorem $1, B$ is weakly compact in $\left(X(B), \gamma_{B}\right)$. Note that the topology $\left.\Im\right|_{X(B)}$ is coarser than the $\gamma_{B}(\cdot)$-topology. It turns out that $B$ is weakly compact in $(X, \Im)$, and we complete the proof. 
Remark. The following two examples indicate that the weak compactness of $B$ and the condition that $\left(X(B), \gamma_{B}\right)^{*}=\left(X(B),\left.\Im\right|_{X(B)}\right)^{*}$ in the last theorem are independent in general.

Example 4. Let $B$ be the closed unit ball of the reflexive Banach space $\left(\ell_{2},\|\cdot\|_{2}\right)$. Let $(X, \Im)=\left(\ell_{2},\|\cdot\|_{\infty}\right)$. Then $\left(X(B), \gamma_{B}\right)=\left(\ell_{2},\|\cdot\|_{2}\right)$ and $B$ is weakly compact in $\left(X(B), \gamma_{B}\right)$. Since the $\|\cdot\|_{\infty}$-topology is coarser than the $\|\cdot\|_{2}$-topology, $B$ is weakly compact in $(X, \Im)$. But

$$
\left(X(B), \gamma_{B}\right)^{*}=\left(\ell_{2},\|\cdot\|_{2}\right)^{*} \neq\left(\ell_{2},\|\cdot\|_{\infty}\right)^{*}=\left(X(B),\left.\Im\right|_{X(B)}\right)^{*}
$$

Example 5. Let $X=\ell_{0}$, the space of finite sequences, and $B$ be the closed unit ball of the normed space $\left(\ell_{0},\|\cdot\|_{\infty}\right)$. Let $\Im$ be the weak topology of $\left(\ell_{0},\|\cdot\|_{\infty}\right)$. Then

$$
\left(X(B), \gamma_{B}\right)^{*}=\left(\ell_{0},\|\cdot\|_{\infty}\right)^{*}=\left(\ell_{0}, \Im\right)^{*}=\left(X(B),\left.\Im\right|_{X(B)}\right)^{*} .
$$

But $B$ is not weakly compact in $(X, \Im)$, since $\left(\ell_{0},\|\cdot\|_{\infty}\right)$ is not reflexive.

\section{A counterexample}

The following example, which is based on a construction of R.C. James [5], may help readers to have a better insight into our conjecture.

Example 6. Let $E$ be a countable real Hilbert product of increasing finite-dimensional $c_{0}$-spaces, so that the members of $E$ are of the form

$$
x=\left(x_{1}^{1} ; x_{1}^{2}, x_{2}^{2} ; x_{1}^{3}, x_{2}^{3}, x_{3}^{3} ; \cdots\right)
$$

with

(1)

$$
\|x\|=\left[\left|x_{1}^{1}\right|^{2}+\left(\sup \left\{\left|x_{1}^{2}\right|,\left|x_{2}^{2}\right|\right\}\right)^{2}+\left(\sup \left\{\left|x_{1}^{3}\right|,\left|x_{2}^{3}\right|,\left|x_{3}^{3}\right|\right\}\right)^{2}+\cdots\right]^{1 / 2}<\infty .
$$

Let $X$ be the linear span of all members $x$ of $E$ such that

$$
\left|x_{1}^{n}\right|=\left|x_{2}^{n}\right|=\cdots=\left|x_{n}^{n}\right| \quad \text { for all } n=1,2, \cdots .
$$

Since $E$ is a Hilbert product of reflexive spaces, $E$ is reflexive. It is easy to see that $X$ is dense in $E$. Note that

$\left.{ }^{*}\right)$ If $x \in X$ and $x$ is a linear combination of $n$ members of $X$ satisfying (2), then for each $m>2^{n}$ at least two of $x_{1}^{m}, \cdots, x_{m}^{m}$ are equal.

Thus the sequence $\left\{\frac{1}{n}\right\}$ belongs to $E$ but not to $X$. Therefore $X \neq E$ and $X$ is not complete. In particular, the closed unit ball $B$ of $X$ is not weakly compact.

We shall verify two facts:

(a) $B$ has James' property (this part is due to R.C. James [5]).

Let $f$ be an arbitrary continuous linear functional on $E$ and $x$ in $E$ be such that $\|x\|=1$ and $f(x)=\|f\|$. Then there is a sequence of numbers $\left(f_{i}^{n}\right)$ such that

$$
f(x)=f_{1}^{1} x_{1}^{1}+\left(f_{1}^{2} x_{1}^{2}+f_{2}^{2} x_{2}^{2}\right)+\left(f_{1}^{3} x_{1}^{3}+f_{2}^{3} x_{2}^{3}+f_{3}^{3} x_{3}^{3}\right)+\cdots .
$$

The norm of $x$ as given by (1) is not changed if for each $n$ we replace each $x_{i}^{n}$ by $\pm \sup _{i}\left|x_{i}^{n}\right|$, where the "+" is used if $f_{i}^{n} \geq 0$ and the "-" if $f_{i}^{n}<0$. The changes $\overline{\mathrm{do}}$ not decrease the sum in (3), so the sum does not change and the new $x$ is a member of the closed unit ball of $X$ at which $f$ attains its supremum.

(b) $B$ does not have the separation property. 
Let

$x=\left(\frac{\sqrt{3}}{2^{n+j-1}}\right)_{j=1, \cdots, n}^{n=1,2,3, \cdots}=\sqrt{3}\left(\frac{1}{2} ; \frac{1}{2^{2}}, \frac{1}{2^{3}} ; \frac{1}{2^{3}}, \frac{1}{2^{4}}, \frac{1}{2^{5}} ; \cdots ; \frac{1}{2^{n}}, \frac{1}{2^{n+1}}, \cdots, \frac{1}{2^{2 n-1}} ; \cdots\right)$.

By $\left(^{*}\right), x \notin X$ and $\|x\|=1$. Let

$$
\begin{aligned}
& x_{1}=\sqrt{3}\left(\frac{1}{2} ; \frac{1}{2^{2}}, \frac{1}{2^{2}} ; \frac{1}{2^{3}}, \frac{1}{2^{3}}, \frac{1}{2^{3}} ; \cdots ; \frac{1}{2^{n}}, \cdots, \frac{1}{2^{n}} ; \cdots\right), \\
& x_{2}=\sqrt{3}\left(\frac{1}{2} ; \frac{1}{2^{2}}, \frac{1}{2^{3}} ; \frac{1}{2^{3}}, \frac{1}{2^{3}}, \frac{1}{2^{3}} ; \cdots ; \frac{1}{2^{n}}, \cdots, \frac{1}{2^{n}} ; \cdots\right), \\
& x_{3}=\sqrt{3}\left(\frac{1}{2} ; \frac{1}{2^{2}}, \frac{1}{2^{3}} ; \frac{1}{2^{3}}, \frac{1}{2^{4}}, \frac{1}{2^{5}} ; \frac{1}{2^{4}}, \frac{1}{2^{4}}, \frac{1}{2^{4}}, \frac{1}{2^{4}} ; \cdots ; \frac{1}{2^{n}}, \cdots, \frac{1}{2^{n}} ; \cdots\right), \\
& \vdots \\
& x_{n}=\sqrt{3}\left(\frac{1}{2} ; \frac{1}{2^{2}}, \frac{1}{2^{3}} ; \frac{1}{2^{3}}, \frac{1}{2^{4}}, \frac{1}{2^{5}} ; \cdots ; \frac{1}{2^{n}}, \frac{1}{2^{n+1}}, \cdots, \frac{1}{2^{2 n-1}} ; \frac{1}{2^{n+1}}, \frac{1}{2^{n+1}}, \cdots, \frac{1}{2^{n+1}} ; \cdots\right) .
\end{aligned}
$$

It is easy to see that $x_{n} \in X$ and $\left\|x_{n}\right\|=1$ for all $n=1,2, \cdots$ and $x_{n} \rightarrow x$ in norm as $n \rightarrow \infty$. Let $a_{n}=\left(1+\frac{1}{n}\right) x_{n}$. It follows that $a_{n} \in X,\left\|a_{n}\right\|=1+\frac{1}{n}$ for all $n=1,2, \cdots$, and $a_{n} \rightarrow x$ in norm as $n \rightarrow \infty$.

Let $A$ be the closed convex hull of the $a_{n}$ 's in $X$. We want to verify that $A$ and the closed unit ball $B$ of $X$ are disjoint. Suppose $y=\left(y_{i}^{m}\right)_{i=1, \cdots, n}^{m=1,2, \cdots}$ is an element of the convex hull of the $a_{n}$ 's. Let $y=\sum_{i=1}^{k} \alpha_{i} a_{n_{i}}$ for some positive integer $k$, where $\alpha_{i} \geq 0$ and $\sum_{i=1}^{k} \alpha_{i}=1$. Then $\|y\|=1+p$, where $p=\sum_{i=1}^{k} \frac{\alpha_{i}}{n_{i}}>0$, and $y_{1}^{m}>y_{2}^{m}>\cdots>y_{m}^{m}$ for $m=1, \cdots, n_{k}$ (without loss of generality, assume $\left.n_{1}<n_{2}<\cdots<n_{k}\right)$. In particular, the convex hull of the $a_{n}$ 's is disjoint from $B$. If $a$ is a cluster point of the convex hull of the $a_{n}$ 's in $E$ with $\|a\|=1$, then $a_{1}^{m}>$ $a_{2}^{m}>\cdots>a_{m}^{m}$ for all $m$. In fact, there exists a sequence $\left\{y_{n}\right\}$ in the convex hull of the $a_{n}$ 's such that $y_{n} \rightarrow a$ as $n \rightarrow \infty$, say $y_{n}=\sum_{i=1}^{k_{n}} \alpha_{i}^{n} a_{i}=\sum_{i=1}^{k_{n}} \alpha_{i}^{n}\left(1+\frac{1}{i}\right) x_{i}$, where $\alpha_{i}^{n} \geq 0, \sum_{i=1}^{k_{n}} \alpha_{i}^{n}=1$, and $k_{n}$ is a positive integer. Then for any positive integer $m, 1 \leq j \leq m$,

$$
\left(y_{n}\right)_{j}^{m}=\frac{\sqrt{3}}{2^{m}}\left(\sum_{i=1}^{m-1} \alpha_{i}^{n}+\sum_{i=1}^{m-1} \frac{\alpha_{i}^{n}}{i}\right)+\frac{\sqrt{3}}{2^{m+j-1}}\left(\sum_{i=m}^{k_{n}} \alpha_{i}^{n}+\sum_{i=m}^{k_{n}} \frac{\alpha_{i}^{n}}{i}\right) .
$$

Now, for any positive integer $m, 1 \leq j \leq m-1$,

$$
\begin{aligned}
a_{j}^{m}-a_{j+1}^{m} & =\lim _{n \rightarrow \infty}\left[\left(y_{n}\right)_{j}^{m}-\left(y_{n}\right)_{j+1}^{m}\right] \\
& =\left(\frac{\sqrt{3}}{2^{m+j-1}}-\frac{\sqrt{3}}{2^{m+j}}\right)\left[\lim _{n \rightarrow \infty}\left(\sum_{i=m}^{k_{n}} \alpha_{i}^{n}+\sum_{i=m}^{k_{n}} \frac{\alpha_{i}^{n}}{i}\right)\right] \\
& \geq\left(\frac{\sqrt{3}}{2^{m+j-1}}-\frac{\sqrt{3}}{2^{m+j}}\right)\left(\lim _{n \rightarrow \infty} \sum_{i=m}^{k_{n}} \alpha_{i}^{n}\right) .
\end{aligned}
$$

If $\lim _{n \rightarrow \infty} \sum_{i=m}^{k_{n}} \alpha_{i}^{n}=0$, then for any given $\varepsilon>0$, there exists a positive integer $M$ such that $\sum_{i=m}^{k_{n}} \alpha_{i}^{n}<\varepsilon$ for all $n \geq M$. This implies $\sum_{i=1}^{m} \alpha_{i}^{n}>1-\varepsilon$ for all $n \geq M$. It follows that for all $n \geq M$,

$$
\left(y_{n}\right)_{j}^{m} \geq \frac{\sqrt{3}}{2^{m}}\left(\sum_{i=1}^{m-1} \alpha_{i}^{n}+\sum_{i=1}^{m-1} \frac{\alpha_{i}^{n}}{i}\right) \geq \frac{\sqrt{3}}{2^{m}}\left(1+\frac{1}{m}\right)\left(\sum_{i=1}^{m-1} \alpha_{i}^{n}\right)>\frac{\sqrt{3}}{2^{m}}\left(1+\frac{1}{m}\right)(1-\varepsilon) .
$$


Therefore

$$
a_{j}^{m}=\lim _{n \rightarrow \infty}\left(y_{n}\right)_{j}^{m} \geq \frac{\sqrt{3}}{2^{m}}\left(1+\frac{1}{m}\right)(1-\varepsilon) .
$$

Let $\varepsilon \rightarrow 0$; we have $a_{j}^{m} \geq \frac{\sqrt{3}}{2^{m}}\left(1+\frac{1}{m}\right)$ for all positive integer $m$ and $1 \leq j \leq m$. Then $\|a\|>1$, which contradicts the fact that $\|a\|=1$. Hence $\lim _{n \rightarrow \infty} \sum_{i=m}^{k_{n}} \alpha_{i}^{n}>0$ and

$$
a_{j}^{m}-a_{j+1}^{m} \geq\left(\frac{\sqrt{3}}{2^{m+j-1}}-\frac{\sqrt{3}}{2^{m+j}}\right)\left(\lim _{n \rightarrow \infty} \sum_{i=m}^{k_{n}} \alpha_{i}^{n}\right)>0
$$

for all positive integers $m$ and $1 \leq j \leq m-1$. By $\left({ }^{*}\right), a \notin X$, and consequently, $a \notin B \subset X$. Hence $A$ and $B$ are disjoint. Next, we show that $A$ and $B$ cannot be strictly separated. Suppose there were a continuous linear functional $f$ of $E$ such that

$$
\sup \{f(b): b \in B\}=\|f\|<\inf \{f(a): a \in A\} .
$$

Since $a_{n} \rightarrow x$ as $n \rightarrow \infty$,

$$
\inf \{f(a): a \in A\} \leq \lim _{n \rightarrow \infty} f\left(a_{n}\right)=f(x) \leq\|f\| .
$$

This is a contradiction! Hence $A$ and $B$ cannot be strictly separated.

\section{Applichtions}

Let us recall that a Banach space is reflexive if and only if its unit ball is weakly sequentially compact [1]. The following extends some of James' results (cf. [4]) from Banach spaces to normed spaces.

Theorem 7. Let $B$ be the closed unit ball of a real normed space $N$. Then the following are equivalent:

(1) $B$ is weakly compact.

(2) B is weakly countably compact.

(3) For each sequence $\left\{x_{n}\right\}$ in $B$ there is an $x$ in $B$ such that for all continuous linear functionals $f$,

$$
\varliminf f\left(x_{n}\right) \leq f(x) \leq \varlimsup \lim f\left(x_{n}\right) .
$$

(4) If $\left\{K_{n}\right\}$ is a decreasing sequence of closed convex sets in $X$ and $B \cap K_{n}$ is non-empty for each $n$, then $B \cap\left(\bigcap_{n \geq 1} K_{n}\right)$ is non-empty.

(5) $B$ is weakly sequentially compact.

(6) If $S$ is a weakly closed set and $B \cap S$ is empty, then $d(B, S)=\inf \{\|b-s\|$ : $b \in B, s \in S\}>0$.

(7) $B$ has the separation property.

Proof. The implications (1) $\Rightarrow(2) \Rightarrow(3) \Rightarrow(4)$ and $(1) \Rightarrow(5) \Rightarrow(6) \Rightarrow(7)$ are proved in [4], and the implication $(7) \Rightarrow(1)$ follows from Theorem 1.

We shall show that $(4) \Rightarrow(7)$. Suppose (4) holds but there were a closed convex set $A$ disjoint from $B$ which cannot be strictly separated from $B$ by a closed hyperplane. In particular, $d(A, B)=0$. We can thus choose $a_{n}$ 's in $A$ and $b_{n}$ 's in $B$ such that $\left\|a_{n}-b_{n}\right\| \rightarrow 0$ as $n \rightarrow \infty$. Let $K_{n}$ be the closed convex hull of $\left\{b_{n}, b_{n+1}, \cdots\right\}$ for $n=1,2, \cdots$. We want to show that $\bigcap_{n \geq 1} K_{n}=\emptyset$. If there exists an element $b$ in $\bigcap_{n \geq 1} K_{n}$, then for all continuous linear functionals $f$, we have

$$
\underline{\lim } f\left(b_{n}\right) \leq f(b) \leq \varlimsup \lim f\left(b_{n}\right) .
$$


As

$$
\left|f\left(a_{n}\right)-f\left(b_{n}\right)\right| \leq\|f\|\left\|a_{n}-b_{n}\right\| \rightarrow 0,
$$

we have

$$
\underline{\lim } f\left(a_{n}\right) \leq f(b) \leq \varlimsup \lim f\left(a_{n}\right), \quad \forall f \in X^{*} .
$$

By the strong separation theorem, $b$ is in the closed convex hull of $\left\{a_{n}, a_{n+1}, \cdots\right\}$ for $n=1,2, \cdots$. Then $b \in A \cap B$. This is a contradiction, and thus $\bigcap_{n \geq 1} K_{n}=\emptyset$. This again conflicts with (4). Hence $B$ has the separation property.

We end this paper with an open problem which seems to be an intermediate (and possibly critical) step to our conjecture.

Problem. Does the continuous linear image of a bounded convex set with the separation property still have the separation property?

It is clear that similar questions concerning weak compactness and James' property have positive answers.

\section{REFERENCES}

1. W. F. Eberlein, Weak compactness in Banach spaces, Proc. Nat. Acad. Sci. U.S.A. 33 (1947), 51-53. MR 9:42; MR 10:855

2. R. C. James, Weakly compact sets, Trans. Amer. Math. Soc. 113 (1964), 129-140. MR 29:2628

3. R. C. James, Weak compactness and separation, Canad. J. Math. 16 (1964), 204-206. MR 28: 455

4. R. C. James, Weakly compactness and reflexivity, Israel J. Math. 2 (1964), 101-119. MR 31:585

5. R. C. James, A counterexample for a sup theorem in normed spaces, Israel J. Math. 9 (1971), 511-512. MR 43:5287

6. R. C. James, Reflexivity and the sup of linear functionals, Israel J. Math. 13 (1972), 289-300. MR 49:3506

7. V. L. Klee, Jr., Convex sets in linear spaces, Duke Math. J., 18 (1951), 443-466, 875-883. MR 13:354,849

8. J. D. Pryce, Weak compactness in locally convex spaces, Proc. Amer. Math. Soc. 17 (1966), 148-155. MR 32:8107

9. H. H. Schaefer, Topological Vector Spaces, Springer-Verlag, New York-Heidelberg-Berlin, 1986. MR 33:1689(earlier ed.)

Department of Applied Mathematics, National Sun Yat-Sen University, Kaohsiung 80424, Tainan, Republic of China

Current address, Hwa-Long Gau: Department of Applied Mathematics, National Chiao Tung University, Hsinchu 300, Taiwan, Republic of China

E-mail address: u8222807@cc.nctu.edu.tw

Department of Applied Mathematics, National Sun Yat-sen University, Kaohsiung 80424, Taiwan, Republic of China

E-mail address: wong@math.nsysu.edu.tw 